\title{
Intravenous morphine titration vs. oral hydrocodone/acetaminophen for adults with lower extremity displaced fracture in an emergency department setting: A randomized controlled trial
}

\author{
ZHENGQI PAN, YONGJIAN QI, YINXIAN WEN and LIAOBIN CHEN \\ Department of Orthopedic Surgery, Zhongnan Hospital of Wuhan University, Wuhan, Hubei 430071, P.R. China
}

Received October 6, 2016; Accepted June 25, 2018

DOI: $10.3892 /$ etm.2018.6606

\begin{abstract}
The objective of the present study was to test the hypothesis that intravenous morphine titration provides superior analgesia to oral hydrocodone/acetaminophen for patients with lower extremity displaced fracture in an emergency department (ED) setting. A prospective, randomized clinical trial of ED patients suffering acute lower extremity displaced fracture pain was performed with a total of 206 participants included. After application of exclusion criteria, the cohort comprised 166 patients, 85 of which were randomly allocated to the oral hydrocodone/acetaminophen $(5 \mathrm{mg} / 500 \mathrm{mg})$ group and 81 to the intravenous morphine titration (every $5 \mathrm{~min}$ by 3 -mg increments) group. The main outcome was the visual analogue scale (VAS) at different time-points after the first dose of analgesic was administered. Secondary outcomes included the VAS change during the skeletal traction operation and short-term adverse events. The results demonstrated that the initial VSA of the participants was similar at the baseline on arrival at the $\mathrm{ED}(\mathrm{P}=0.2582)$. At the time-points of $5,15,30 \mathrm{~min}$ after the first dose of analgesic administered, the intravenous morphine titration group exhibited a greater VAS reduction compared with that in the oral hydrocodone/acetaminophen group $(\mathrm{P}<0.01)$. The differences between the 2 groups were not statistically significant at $1 \mathrm{~h}$ or thereafter. The incidence of short-term adverse events was similar between the 2 groups but sedation, whose incidence in the morphine group was markedly increased, may not be arbitrarily attributed to adverse events. It was concluded that, compared with oral hydrocodone/acetaminophen, intravenous morphine titration provided a rapid and sufficient pain relief
\end{abstract}

Correspondence to: Professor Liaobin Chen, Department of Orthopedic Surgery, Zhongnan Hospital of Wuhan University, 169 East Lake Road, Wuhan, Hubei 430071, P.R. China

E-mail: lbchen@whu.edu.cn

Key words: oral hydrocodone/acetaminophen, intravenous morphine titration, analgesia, lower extremity displaced fracture, emergency department and equivalent short-term adverse events for patients with lower extremity displaced fracture in an ED setting.

\section{Introduction}

Moderate to severe pain is a particularly outstanding complaint for patients with lower extremity displaced fracture in an emergency department (ED) setting. This traumatic experience is exaggerated by certain necessary interventions such as transportation, radiographic examination and skeletal traction (1). Adequate analgesia is considered a fundamental component of patient care at the ED and may improve patient satisfaction metrics and medical experience (2), while insufficient analgesia has been reported to cause short-term problems such as anxiety, hyperesthesia and fear of medical care (3). There is a general consensus that analgesia should be one of the primary targets of any treatment setting in the ED. The ability to deliver analgesics in a short time window is one of the most important missions for any emergency physician.

As an analgesic with central effects, acetaminophen elevates the pain threshold in the central nervous system. It has been used effectively in relieving moderate to severe pain arising from events such as renal colic (4) and cesarean section (5). It has also been widely used for acute traumatic pain relief in the ED setting (6). Hydrocodone, a semisynthetic opioid, has a good pharmacokinetic profile with a high oral bioavailability. It has been identified to exert a synergistic analgesic effect with acetaminophen in numerous studies $(7,8)$. Due to its convenient mode of delivery, oral hydrocodone/acetaminophen is the analgesic most commonly prescribed by emergency physicians (9). The majority of studies observed that the hydrocodone/acetaminophen combination exerts its analgesic effect in $30 \mathrm{~min}$ and beyond $(10,11)$, which means that the onset time is not fast enough, particularly for fracture patients with violent pain.

As a strong opioid drug, morphine has served as a favorable analgesic in the ED setting for decades. Intravenous morphine has been widely used for severe pain control for conditions such as drastic pain with acute myocardial infarction (12) and terminal cancer (13). In addition, short-term intravenous morphine administration provided a lighter sedation to relieve the patients' anxiety (14). The 
use of an intravenous morphine titration provided a rapid and sufficient pain relief under the minimum effective dose with the least amount of adverse effects (15). It was reported that the onset time of intravenous morphine titration may be as short as $3 \mathrm{~min}$ with most of the pain relieved during acute pain management but without any obvious severe adverse events (15-17). It was suggested that intravenous morphine titration may be used as a reasonable analgesic for moderate to severe pain in the ED, particularly due to its extremely short onset time and sufficient analgesic efficiency.

The objective of the present study was to compare the analgesic efficacy and adverse events of hydrocodone/acetaminophen vs. intravenous morphine titration for moderate to severe pain relief in patients with lower extremity displaced fracture at the ED. It was hypothesized that intravenous morphine titration provides more rapid and efficient pain relief than oral hydrocodone/acetaminophen in an ED setting.

\section{Materials and methods}

Study design. The procedure of the present randomized controlled clinical trial was in accordance with the Declaration of Helsinki from 1975 and its revision from 2000. The protocol was approved by the ethics committee of Zhongnan Hospital of Wuhan University (Wuhan, China). The participants were recruited from the ED of Zhongnan Hospital, Wuhan University (Wuhan, China). An approximate annual census of 80,000 patients is treated in this department each year, of which $>800$ are lower extremity displaced fractures. All participants or their legal representative provided written informed consent prior to enrollment. The time-period for the case collection was from September 2014 to September 2015.

Sample size. The sample size was calculated to achieve an alpha of 0.05 and a statistical power of $80 \%$, leading to an estimate of at least 73 patients per group being required (10). Due to the uncertainty of these assumptions, it was decided to allow a large error margin by aiming to recruit $>80$ patients per group.

Participants. Healthy adults who were $\geq 18$ and $\leq 65$ years of age were considered eligible if they had a suspected lower extremity displaced fracture within $2 \mathrm{~h}$ after trauma. The included participants had moderate or severe pain [visual analogue scale (VAS) $\geq 70$ ] with a pulse oxygen saturation of $\geq 95 \%$ and a respiratory rate of $\geq 12$ breaths $/ \mathrm{min}$. Exclusion criteria were as follows: Pregnancy or lactation; chronic hepatic or renal disease; multiple injuries with unstable hemodynamics; pathological fracture; dementia or delirium and inability to understand the pain scales; history of prior or current analgesia use; allergy to acetaminophen, hydrocodone or morphine. Due to insufficient pain intensity in most cases, the patients with foot fracture were also excluded.

Study protocol. Patients who presented at the ED with a tentative diagnosis of a lower extremity displaced fracture and who met the inclusion criteria were approached for consent to enter the study. They were randomized into one of two groups. The baseline physiological parameters were collected:
Blood pressure, pulse, pulse oxygen saturation, respiratory rate and the initial VAS. The following dynamic VAS data were obtained at the time-points of 5, 15, 30, 60 and 120 min after the first dose of analgesic drug administered. Any adverse events were recorded. When the patients underwent skeletal traction, the VAS prior to and during the skeletal traction operation was also recorded.

After providing consent, the participants eligible for the study were randomly assigned to treatment with oral hydrocodone/acetaminophen or intravenous morphine titration. In the oral hydrocodone/acetaminophen group, the dose of the tablet was $5 \mathrm{mg} / 500 \mathrm{mg}$ (18), and only one dose was given. The method of intravenous administration was reported in a previous study (17). In brief, after multifunctional monitoring and venous channel establishing, morphine was titrated every $5 \mathrm{~min}$ by $3-\mathrm{mg}$ increments and pain was evaluated every $5 \mathrm{~min}$ until substantial partial remission. Intravenous morphine titration was terminated if the patient presented with one of the following conditions: VAS, $\leq 60$; respiratory rate, $\leq 12$; pulse oxygen saturation, $\leq 95 \%$; or a severe adverse event associated with morphine administration (respiratory depression or hyperemesis).

Outcome. The main outcome measure was the difference in the mean VAS between the groups at each time-point. The secondary outcome included the VAS change during the skeletal traction operation and short-term adverse events.

Statistical analysis. SPSS version 18 (SPSS Inc., Chicago, IL, USA) was used for statistical analysis. Data were analyzed using Student's t-test for continuous variables and Pearson's $\chi^{2}$ test for categorical variables. $\mathrm{P}<0.05$ was considered to indicate a statistically significant difference.

\section{Results}

Patient characteristics. The flow diagram for enrolment of the subjects in the present trial is presented in Fig. 1 and baseline characteristics are displayed in the Table I. During the 1-year study period, a total of 206 patients who presented with lower extremity displaced fracture and a VAS of $>70$ were enrolled in the study, of which 166 were eligible for analysis. The most common reason for exclusion was multiple trauma, which accounted for nearly one third of all excluded patients. Of these, 85 and 81 patients were randomized into the hydrocodone/acetaminophen group and morphine group, respectively. The number of patients in each group available for analysis exceeded the pre-specified sample size requirements. There were no statistically significant differences in baseline variables between the 2 groups, including age, sex distribution, body mass index, fracture sites and time since injury. The initial VAS prior to pain medication on arrival to the ED was similar in the two groups.

Effects of analgesic treatments on VAS. The onset time and pain relief degree were the main indices to estimate the analgesic efficiency of each analgesic treatment. The present study evaluated the VAS at several time-points, starting from as soon as $5 \mathrm{~min}$ after the first dose administration (Fig. 2). It was observed that the intravenous morphine titration group 


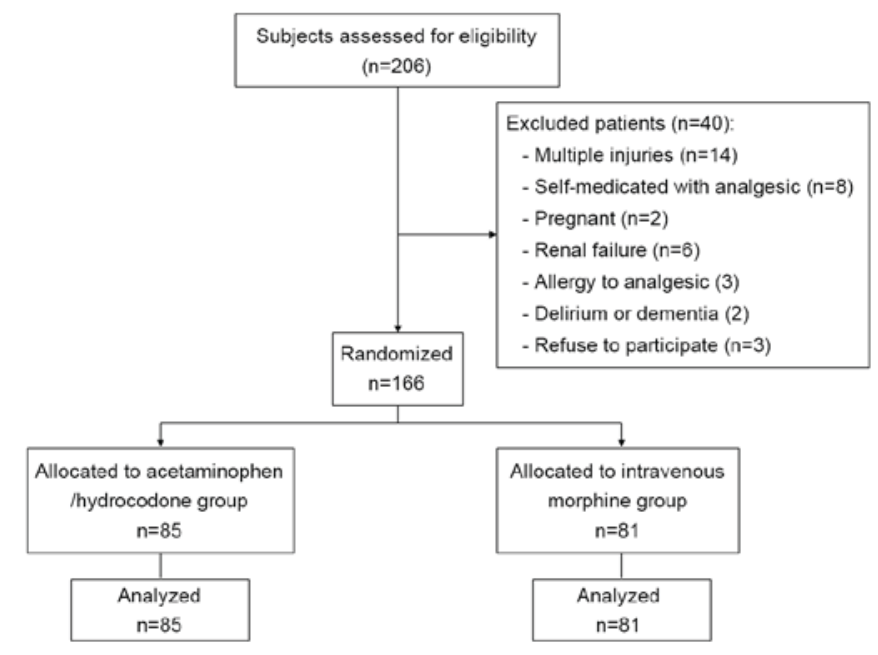

Figure 1. Flow diagram illustrating the selection of subjects for enrolment in the trial.

had a greater VAS reduction compared with that in the oral hydrocodone/acetaminophen group at the time-points of 5 , $15,30 \mathrm{~min}$ after the first analgesic administration $(\mathrm{P}<0.01$ for each). The differences were not statistically significant between the 2 groups at the time-point of $1 \mathrm{~h}$ and beyond. It was speculated that intravenous morphine titration may bring a more rapid pain reduction than oral hydrocodone/acetaminophen for moderate to severe pain in patients with lower extremity displaced fracture.

The vast majority of lower extremity displaced fractures require skeletal traction contributing to temporary fracture reduction, which invokes pain during the operation due to the extruding of fracture ends. Therefore, the analgesic effect prior to and during the skeletal traction operation was evaluated. As presented in Fig. 3, the VAS did not significantly increase in the intravenous morphine titration group, while it was significantly elevated in the oral hydrocodone/acetaminophen group. This suggested that intravenous morphine titration provided consistent analgesia even during movement.

Adverse events. Short-term adverse events were among the greatest concerns of the ED physicians and patients, particularly apparent severe adverse effects such as severe ventilatory depression. The adverse events are tabulated in Table II. There were no apparent severe adverse events observed in the present study. The most frequently observed adverse event was nausea (17.6\% in the hydrocodone/acetaminophen vs. $13.6 \%$ in the morphine group). The incidence of nausea, pruritus, dizziness and vomiting was similar between the two groups. However, the incidence of sedation in the morphine group was significantly higher.

\section{Discussion}

Achieving rapid pain relief is among the most desired factors in patients with displaced fractures presenting at the ED (19). Delay in pain relief by even one minute worsens the medical experience, while it is also associated with potential complications such as increased risk of cardiovascular events (18). American guidelines for pain management in the ED recommend the prioritization of pain relief, with administration of analgesics performed even prior to the establishment of an etiology (20). The present study compared 2 analgesics frequently used at the ED for patients with lower extremity displaced fractures, who experienced moderate to severe pain. It was found that compared with oral hydrocodone/acetaminophen, intravenous morphine titration provided a more rapid and efficient pain relief without any apparent short-term severe adverse effects. The onset time was even shortened to $5 \mathrm{~min}$ after the first morphine bolus dose.

With rapid and efficient analgesia, the patients are comfortable and have a more positive medical experience while waiting for a further evaluation and therapy at the ED (11). In addition, the length of the stay at the ED may be shorter if patients receive adequate analgesia (21). A variety of analgesics are applied to fracture patients at the ED. Depending on their mode of application and mechanism of action, frequently-used analgesics at the ED include non-steroidal anti-inflammatory drugs (NSAIDS) (10), opiates (22), narcotics (23), regional anesthesia (e.g. fascia iliaca compartment block) (24) and even Traditional Chinese Medicine (e.g. acupuncture) (25). Among these analgesics, narcotics such as fentanyl or remifentanil have the shortest onset time with $2.6 \pm 1.8 \mathrm{~min}$ (23). However, access to intravenous narcotics is difficult due to overly bureaucratic restrictions in developing countries, particularly at EDs (26). NSAIDs such as ibuprofen are usually used to control mild to moderate pain (27). Regional anesthesia and Traditional Chinese Medicine may be challenging for ED physicians to master due to their specialized application. In order to maximize the analgesic effect and minimize adverse events, the combination of different analgesics applied at the right moment is of advantage, with oral hydrocodone/acetaminophen being the most frequently prescribed (18). Similar to the results of the present study, the majority of previous studies demonstrated that the onset time was 1-2 h, which hardly satisfied the time effectiveness required at the $\operatorname{ED}(8,18)$. As a commonly used analgesic at the ED, intravenous morphine was reported to have a shorter analgesic onset time than other methods (28). As observed in the present study, the onset time in the morphine group was shortened to $5 \mathrm{~min}$ within administration of the first dose. It is therefore recommended that intravenous morphine is used as an analgesic of choice for patients with lower extremity displaced fracture.

Patient control analgesia (PCA) has gradually turned into the gold standard of pain management and has been widely used for relieving acute pain at the ED (29). PCA has been reported to provide effective pain relief with less analgesic consumption but greater patient satisfaction when compared with other analgesic administration methods, including oral and intravenous bolus (15). In fact, the mode of delivery of morphine by intravenous titration was similar to PCA with the controlling device located in hands of the physicians or nurses. Compared with oral delivery method, intravenous titration did not significantly increase the workload of medical providers $(15,30)$. In accordance with the results of numerous other studies $(15,30)$, the present study demonstrated that intravenous morphine titration provided rapid and sufficient relief for moderate to severe pain.

Displacement of the fracture ends may aggravate the acute pain, and in turn, the muscle spasm caused by acute pain may accentuate the displacement, leading to a vicious cycle of pain amplification (31). The frequent movement during interventions 
Table I. General characteristics of participants.

\begin{tabular}{|c|c|c|c|}
\hline Characteristics & Acetaminophen/hydrocodone group $(n=85)$ & Morphine group $(\mathrm{n}=81)$ & P-value \\
\hline Age (years) & $45.2 \pm 8.7$ & $43.8 \pm 8.2$ & 0.2281 \\
\hline Female sex & $24(23.53)$ & $19(23.46)$ & 0.5994 \\
\hline BMI (kg) & $26.3 \pm 4.1$ & $25.8 \pm 4.5$ & 0.3998 \\
\hline \multicolumn{4}{|l|}{ Site of fracture } \\
\hline Femur & $44(51.74)$ & $36(44.44)$ & 0.4306 \\
\hline Tibia & $41(48.26)$ & $45(55.56)$ & 0.4306 \\
\hline Initial VAS & $88.0 \pm 6.8$ & $89.1 \pm 5.6$ & 0.0815 \\
\hline
\end{tabular}

Values are expressed as the mean \pm standard deviation or $\mathrm{n}(\%)$. BMI, body mass index; VAS, visual analogue scale.

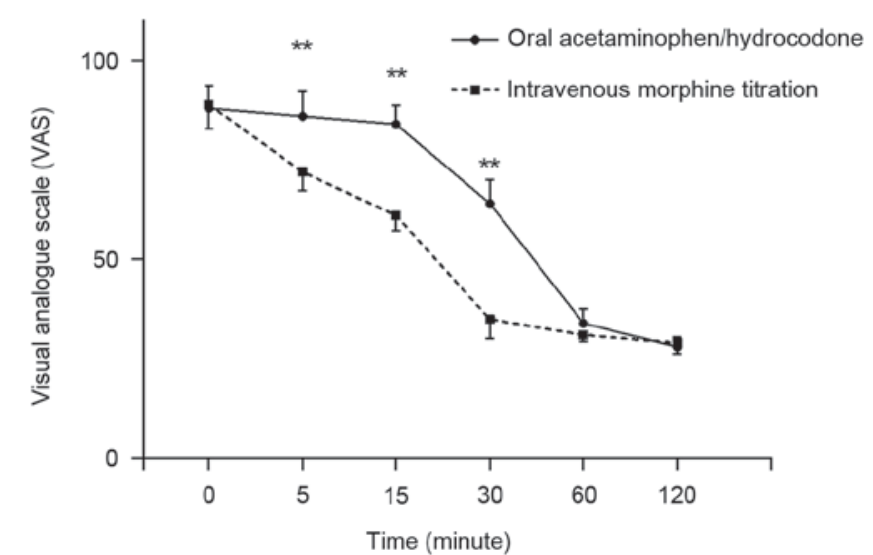

Figure 2. VAS of the oral hydrocodone/acetaminophen and intravenous morphine titration group at each time-point. Values are expressed as the mean \pm standard deviation. ${ }^{* * *} \mathrm{P}<0.01$ for comparison between the two groups. VAS, visual analogue scale.

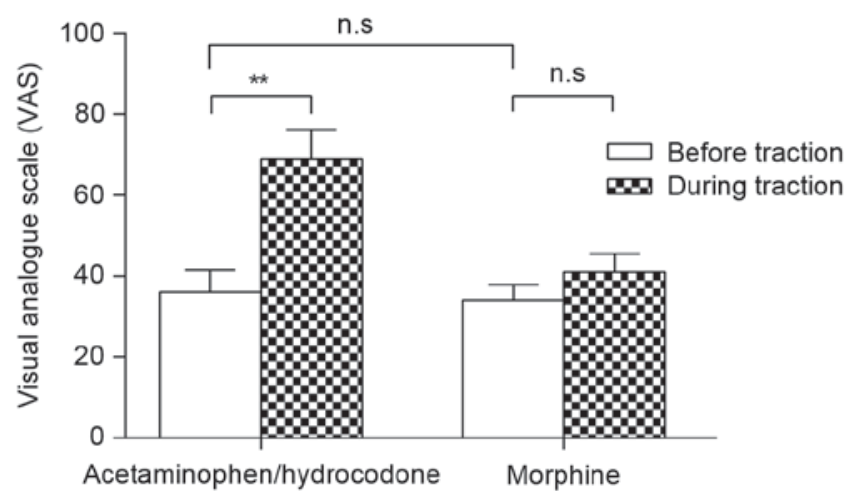

Figure 3. VAS of the oral hydrocodone/acetaminophen and intravenous morphine titration group prior to and during the skeletal traction operation. Values are expressed as the mean \pm standard deviation. ${ }^{* *} \mathrm{P}<0.01$ as indicated. VAS, visual analogue scale; n.s., no significance.

such as radiological examinations and traction application may provoke the pain derived from the friction of the fracture ends (32). Therefore, analgesia is necessary to alleviate the pain during these procedures. Regional anesthesia techniques such as femoral nerve or fascia iliaca block, may be employed to avoid adverse events compared with those of analgesics, but their use at the ED may be limited due to their complex operation (24). Of
Table II. Short-term adverse events in the acetaminophen/ hydrocodone group $(\mathrm{n}=85)$ and the morphine group $(\mathrm{n}=81)$.

\begin{tabular}{lccc}
\hline $\begin{array}{l}\text { Adverse } \\
\text { event }\end{array}$ & $\begin{array}{c}\text { Acetaminophen/ } \\
\text { hydrocodone, } \mathrm{n}(\%)\end{array}$ & $\begin{array}{c}\text { Morphine, } \\
\mathrm{n}(\%)\end{array}$ & P-value \\
\hline Nausea & $15(17.6)$ & $11(13.6)$ & 0.6162 \\
Pruritus & $8(9.4)$ & $6(7.4)$ & 0.8531 \\
Dizziness & $9(10.6)$ & $6(7.4)$ & 0.6572 \\
Vomiting & $9(10.6)$ & $13(16.0)$ & 0.4189 \\
Sedation & $4(4.7)$ & $13(16.0)$ & 0.0355
\end{tabular}

Certain patients had $>1$ adverse event.

note, the VAS was not markedly increased during the skeletal traction operation following the intravenous morphine titration, while oral hydrocodone/acetaminophen was not able to maintain a stable VAS during traction operation, which demonstrated that intravenous morphine titration provided consistent pain relief not only in the resting but also in the movement state.

In the present study, no severe short-term adverse events were observed, but pruritus, nausea and dizziness, which are the most common side effects of opioids, occurred with a comparable incidence. Compared with oral hydrocodone/acetaminophen, intravenous morphine titration significantly increased the incidence of sedation. This adverse event brought no further damage and were improved after timely routine treatments. It is important to note that morphine-induced sedation may be beneficial to relieve anxiety generated by acute pain and may not be arbitrarily attributed to adverse events. In addition, in cases of severe respiratory depression (respiratory rate, $<10$ per minute), intravenous naloxone $(0.04 \mathrm{mg})$ and transitory mechanical ventilation was administered until the risk is eliminated (33), indicating that intravenous morphine titration is safe in an ED setting.

Of note, the present study had certain limitations. First, a constant dose of hydrocodone/acetaminophen was used in all participants. In fact, several different dosages, such as $325 \mathrm{mg} / 5 \mathrm{mg}, 600 \mathrm{mg} / 5 \mathrm{mg}$ and $600 \mathrm{mg} / 7.5 \mathrm{mg}$, were prescribed by physicians in ED settings according to previous studies $(8,18,34)$. Furthermore, as analgesia was performed in the specific ED setting, the present short-term observation 
did not provide any evidence with regard to the efficacy and safety on a long-term basis. In addition, further studies may be performed in other populations (e.g. children and elderly patients) with other sources of pain. Finally, owing to limited time and funding, no larger sample size was achieved in spite of the availability of an adequate amount of eligible participants.

In conclusion, compared with oral hydrocodone/acetaminophen, intravenous morphine titration may provide a sufficient and more rapid pain relief and equivalent short-term adverse events for patients with moderate and severe pain. As an ideal analgesia, intravenous morphine titration may be recommended for patients with lower extremity displaced fracture in an ED setting.

\section{Acknowledgements}

Not applicable.

\section{Funding}

The present study was supported by grants from the National Natural Science Foundation of China (grant no. 81371940) and Zhongnan Hospital of Wuhan University Science, Technology and Innovation Seed Fund (grant no. cxpy2017021).

\section{Availability of data and materials}

The datasets used and/or analyzed during the current study are available from the corresponding author on reasonable request.

\section{Authors' contributions}

$\mathrm{ZP}$ and LC designed and directed the experiments; YQ and YW performed the statistical analysis of data; ZP wrote the manuscript; and ZP, YQ and YW reviewed and edited the manuscript. All authors read and approved the final manuscript.

\section{Ethics approval and consent to participate}

The protocol was approved by the Ethics Committee of Zhongnan Hospital of Wuhan University (Wuhan, China) and all participants or their legal representative provided written informed consent prior to enrollment.

\section{Patient consent for publication}

All patients provided written informed consent for research and publication.

\section{Competing interests}

The authors declare that they have no competing interests.

\section{References}

1. Hosseinzadeh Nik T, Shahsavari N, Ghadirian H and Ostad SN Acetaminophen versus liquefied ibuprofen for control of pain during separation in orthodontic patients: A randomized triple blinded clinical trial. Acta Med Iran 54: 418-421, 2016.

2. Wattana MK and Todd KH: Patient satisfaction surveys and treatment of pain in the emergency department (ED) setting. Pain Med 14: 968-969, 2013.
3. Kapoor S, White J, Thorn BE and Block P: Patients presenting to the emergency department with acute pain: The significant role of pain catastrophizing and state anxiety. Pain Med 17: 1069-1078, 2016.

4. Kaynar M, Koyuncu F, Buldu I, Tekinarslan E, Tepeler A, Karatağ T, İstanbulluoğlu MO and Ceylan K: Comparison of the efficacy of diclofenac, acupuncture, and acetaminophen in the treatment of renal colic. Am J Emerg Med 33: 749-753, 2015.

5. Valentine AR, Carvalho B, Lazo TA and Riley ET: Scheduled acetaminophen with as-needed opioids compared to as-needed acetaminophen plus opioids for post-cesarean pain management. Int J Obstet Anesth 24: 210-216, 2015.

6. Pickering G, Moustafa F, Macian N, Schmidt J, Pereira B and Dubray C: A new transmucous-buccal formulation of acetaminophen for acute traumatic pain: A non-inferiority, randomized, double-blind, clinical trial. Pain Physician 18: 249-257, 2015.

7. Hewitt DJ, Todd KH, Xiang J, Jordan DM and Rosenthal NR; CAPSS-216 Study Investigators: Tramadol/acetaminophen or hydrocodone/acetaminophen for the treatment of ankle sprain: A randomized, placebo-controlled trial. Ann Emerg Med 49: 468-480, 480.e1-2, 2007.

8. Chang AK, Bijur PE, Munjal KG and John Gallagher E: Randomized clinical trial of hydrocodone/acetaminophen versus codeine/acetaminophen in the treatment of acute extremity pain after emergency department discharge. Acad Emerg Med 21: 227-235, 2014.

9. Niska R, Bhuiya F and Xu J: National hospital ambulatory medical care survey: 2007 emergency department summary. Natl Health Stat Report: 1-31, 2010.

10. Graudins A, Meek R, Parkinson J, Egerton-Warburton D and Meyer A: A randomised controlled trial of paracetamol and ibuprofen with or without codeine or oxycodone as initial analgesia for adults with moderate pain from limb injury. Emerg Med Australas 28: 666-672, 2016.

11. Drendel AL, Gorelick MH, Weisman SJ, Lyon R, Brousseau DC and Kim MK: A randomized clinical trial of ibuprofen versus acetaminophen with codeine for acute pediatric arm fracture pain. Ann Emerg Med 54: 553-560, 2009.

12. McCarthy CP, Mullins KV, Sidhu SS, Schulman SP and McEvoy JW: The on- and off-target effects of morphine in acute coronary syndrome: A narrative review. Am Heart J 176: 114-121, 2016.

13. Zeppetella G: Opioids for the management of breakthrough cancer pain in adults: A systematic review undertaken as part of an EPCRC opioid guidelines project. Palliat Med 25: 516-524, 2011.

14. Carrer S, Bocchi A, Candini M, Donegà L and Tartari S: Short term analgesia based sedation in the Intensive Care Unit: Morphine vs remifentanil + morphine. Minerva Anestesiol 73: 327-332, 2007.

15. Rahman NH and DeSilva T: The effectiveness of patient control analgesia in the treatment of acute traumatic pain in the emergency department: A randomized controlled trial. Eur J Emerg Med 19: 241-245, 2012.

16. Lvovschi V, Aubrun F, Bonnet P, Bouchara A, Bendahou M, Humbert B, Hausfater P and Riou B: Intravenous morphine titration to treat severe pain in the ED. Am J Emerg Med 26: 676-682, 2008.

17. Pan Z, Qi Y, Wen Y and Chen L: Intravenous morphine titration as a rapid and efficient analgesia for adult patients with femoral shaft fractures after injury. Am J Emerg Med 34: 2107-2111, 2016.

18. Chang AK, Bijur PE, Holden L and Gallagher EJ: Comparative analgesic efficacy of oxycodone/acetaminophen versus hydrocodone/acetaminophen for short-term pain management in adults following ED discharge. Acad Emerg Med 22: 1254-1260, 2015.

19. Deaton T, Auten JD and Darracq MA: Nebulized fentanyl vs intravenous morphine for ED patients with acute abdominal pain: A randomized double-blinded, placebo-controlled clinical trial. Am J Emerg Med 33: 791-795, 2015.

20. Patrick PA, Rosenthal BM, Iezzi CA and Brand DA: Timely pain management in the emergency department. J Emerg Med 48: 267-273, 2015.

21. Kavanagh PL, Sprinz PG, Wolfgang TL, Killius K, Champigny M, Sobota A, Dorfman D, Barry K, Miner R and Moses JM: Improving the management of vaso-occlusive episodes in the pediatric emergency department. Pediatrics 136: e1016-e1025, 2015. 
22. Neven D, Paulozzi L, Howell D, McPherson S, Murphy SM, Grohs B, Marsh L, Lederhos C and Roll J: A randomized controlled trial of a citywide emergency department care coordination program to reduce prescription opioid related emergency department visits. J Emerg Med 51: 498-507, 2016.

23. Gharavifard M, Tafakori A and Zamani Moghadam $\mathrm{H}$ : Remifentanil versus fentanyl/midazolam in painless reduction of anterior shoulder dislocation; a randomized clinical trial. Emerg (Tehran) 4: 92-96, 2016.

24. Høgh A, Dremstrup L, Jensen SS and Lindholt J: Fascia iliaca compartment block performed by junior registrars as a supplement to pre-operative analgesia for patients with hip fracture. Strategies Trauma Limb Reconstr 3: 65-70, 2008.

25. Tsai SL, Fox LM, Murakami M and Tsung JW: Auricular acupuncture in emergency department treatment of acute pain. Ann Emerg Med 68: 583-585, 2016.

26. Berterame S, Erthal J, Thomas J, Fellner S, Vosse B, Clare P, Hao W, Johnson DT, Mohar A, Pavadia J, et al: Use of and barriers to access to opioid analgesics: A worldwide, regional, and national study. Lancet 387: 1644-1656, 2016.

27. Shepherd M and Aickin R: Paracetamol versus ibuprofen: A randomized controlled trial of outpatient analgesia efficacy for paediatric acute limb fractures. Emerg Med Australas 21: 484-490, 2009.

28. Abou Hammoud H, Simon N, Urien S, Riou B, Lechat P and Aubrun F: Intravenous morphine titration in immediate postoperative pain management: Population kinetic-pharmacodynamic and logistic regression analysis. Pain 144: 139-146, 2009.
29. Rahman NH and DeSilva T: A randomized controlled trial of patient-controlled analgesia compared with boluses of analgesia for the control of acute traumatic pain in the emergency department. J Emerg Med 43: 951-957, 2012.

30. Chang AK, Bijur PE, Davitt M and Gallagher EJ: Randomized clinical trial of an intravenous hydromorphone titration protocol versus usual care for management of acute pain in older emergency department patients. Drugs Aging 30: 747-754, 2013.

31. Joshi N, Lira A, Mehta N, Paladino L and Sinert R: Diagnostic accuracy of history, physical examination, and bedside ultrasound for diagnosis of extremity fractures in the emergency department: A systematic review. Acad Emerg Med 20: 1-15, 2013.

32. Bumpass DB, Ricci WM, McAndrew CM and Gardner MJ: A prospective study of pain reduction and knee dysfunction comparing femoral skeletal traction and splinting in adult trauma patients. J Orthop Trauma 29: 112-118, 2015.

33. Patanwala AE, Keim SM and Erstad BL: Intravenous opioids for severe acute pain in the emergency department. Ann Pharmacother 44: 1800-1809, 2010.

34. Turturro MA, Paris PM and Larkin GL: Tramadol versus hydrocodone-acetaminophen in acute musculoskeletal pain: A randomized, double-blind clinical trial. Ann Emerg Med 32: 139-143, 1998. 